\title{
Image or identity? Only Super-Recognizers' (memor)ability is consistently viewpoint-invariant
}

\author{
Jeffrey D Nador ${ }^{1^{*}}$, Tamara A Alsheimer ${ }^{1,2^{*}}$, Ayla Gay ${ }^{1}$ \& Meike Ramon ${ }^{\bullet}$ \\ ${ }^{1}$ Applied Face Cognition Lab, University of Fribourg, Fribourg, Switzerland; 2Experimental Psychology, Justus-Leibig University, Gießen, \\ Germany; *Shared authorship; •Correspondence: meike.ramon@gmail.com
}

A face's memorability refers to the unique combination of its intrinsic visual features facilitating its later recognition. Despite considerable variation in face recognition ability amongst the general population, individuals show substantial concordance regarding the memorability of various faces. And, when the viewpoints across which identities are seen at encoding and recognition differ, such agreement persists, though to a lesser extent. Consequently, face recognition cannot rely solely on image-dependent encoding; individuals must extract some invariant facial information, robust to changes in viewpoint, to do so consistently. However, whether such consistency covaries with overall face processing ability is unclear. Here, therefore, in two experiments we tested recognition of (i) implicitly encoded face images and (ii) explicitly encoded identities in a group of normal control observers against a group of "Super-Recognizers" (SRs) who possess exceptional face processing skills. When implicit encoding was surreptitiously solicited, recognition of studied images was comparable between groups. Yet, when encoding was explicitly solicited, SRs more accurately recognized studied identities across viewpoint changes than normal observers. Critically, imagedependent information could only inform recognition in the first experiment, whereas viewpoint-invariant information could inform recognition consistently in both. Individualized profiles of observers' performance (as a function of stimulus memorability) reveal that only SRs performed consistently between experiments. We suggest that SRs' unique capacity for utilizing viewpoint-invariant information for recognition, regardless of encoding conditions, is rooted in fundamentally more accurate and robust representations of identity-based memorability. These results invite a reinterpretation of face memorability that describes viewpoint-invariant information, diagnostic of facial identity representations in memory.

Keywords: Memorability; Super-Recognizers; Face recognition; Individual differences; Consistency; Pattern analysis.

\section{Introduction}

Normally, we recognize and identify others based on a variety of aspects of their appearance; their clothing, hair style, gait, and posture all serve as potential cues revealing their identity. But, maybe most critically of all, we recognize and identify individuals based on facial information. And yet, while some people's faces may be readily recognizable to us, others may be just as forgettable.

A face's memorability refers to the combination of its intrinsic visual features tending to facilitate its later recognition, which is distinct from its saliency or other common priming 
effects (Bainbridge et al., 2017; Bainbridge, 2020; see Rust \& Mehrpour, 2020 for review)1. Indeed, a growing body of literature supports the idea that faces (and other objects) are not all equally memorable: there is inter-individual consistency between the memorability of specific images, and of identities (Isola et al., 2011; Bainbridge et al., 2013; Bainbridge, 2017, 2020). That is, the faces we recognize tend to converge with those recognized by others, and viceversa (Bainbridge, 2017; Vokey \& Read; 1992; Rust \& Mehrpour, 2020). As a whole, the extant literature suggests that a face's memorability is an intrinsic property derivable from some combination of two types on information: image-dependent information (i.e., imagespecific, low-level visual features), as well as viewpoint-invariant information (i.e., cues persisting across images of the same identity) (Bainbridge, 2017; Chang et al., 2017).

The implication is that this particular combination ought to be quantifiable along some theoretical stimulus dimension (or some higher dimensional space). So, while face images can be reconstructed in a "face space" from similarity ratings between face images (reflecting image-dependent similarities), or face images and memorial representations (reflecting viewpoint-invariant similarities) (Chang et al., 2017), image statistics alone are insufficient for quantifying memorability either in human observers, or using machine learning algorithms limited to pixel-wise matching (Isola et al, 2011; Chang et al., 2017). Therefore, it is not surprising, from the standpoint of human observers, that identity recognition cannot be achieved based on image statics alone.

This raises an interesting question: What are the relative contributions of image- and identitybased memorability to face recognition more broadly? Image recognition demands highly accurate perception, whereas recognizing facial identities poses the additional requirement of constructing invariant representations that translate across changing viewpoints and environmental conditions. Thus, while recognizing specific images could be accomplished using either image or identity information, doing so consistently across changes in viewpoint necessarily requires both to be successful.

Here, to address the above question, we capitalized on the extraordinary face processing abilities of so-called SRs (Isola et al., 2011; Ramon et al., 2019; Ramon, In press) to characterize plausible differential reliance on image-dependent and viewpoint-invariant information between these individuals and typical observers, as a function of memorability. We investigated whether SRs would show greater sensitivity to either type of information by assessing their recognition of specific face images, and facial identities captured from different viewpoints, respectively. Importantly, we implemented a novel "With or without you" (WoWY) analytical approach that departs fundamentally from previous work both on SRs and visual memorability. Past studies have focused on aggregate scores that gloss over latent variability in memorability (both within experiments, and across studies). We reason instead that memorability constitutes an ideal construct for probing the internal consistency of image and

\footnotetext{
1 The umbrella term memorability congregates behavioral recognition performance measured in vastly different ways, most importantly across substantially different time scales and under different conditions (Bainbridge, 2020). For instance, Bainbridge (2017) determined the memorability of stimuli in the context of an 'Identity Memory Game' in which face images were displayed sequentially, and observers responded with a button press whenever they thought an identity repeated, thereby resembling an $n$ back task. Here, we split the study and recognition of faces into separate phases of each experiment, measuring memorability over a slightly longer delay. Others have used increased delays and a prolonged study phase to examine long-term memorability (see e.g., Rugo et al., 2017). Note that the term recognition refers to the ability to discern previously encountered as opposed to novel, unseen stimuli, as in the context of old/new recognition tasks employed here. This is not to be confused with identification, which requires additional retrieval of identity-specific semantic information associated with individuals (Ramon \& Gobbini, 2018; Ramon, 2018; Fysh et al, 2020; Stacchi et al., 2020; Ramon, 2021).
} 
identity recognition, which in turn may directly correspond to individual differences in observers' idiosyncratic capacities.

In Experiment 1, we surreptitiously tested recognition for (previously memorability ranked, c.f. Bainbridge et al., 2013) face images (Figure 1, top): after initial implicit learning (during a gender categorization task) the exact same images were-without warning-presented as targets among novel distractors in an old/new recognition task. At base, we hypothesized that recognition performance would be greater for high versus low memorability face images. Moreover, if SRs were to show greater sensitivity to memorability than controls, this would suggest that they have generally superior memory for face images.

\section{Experiment 1}

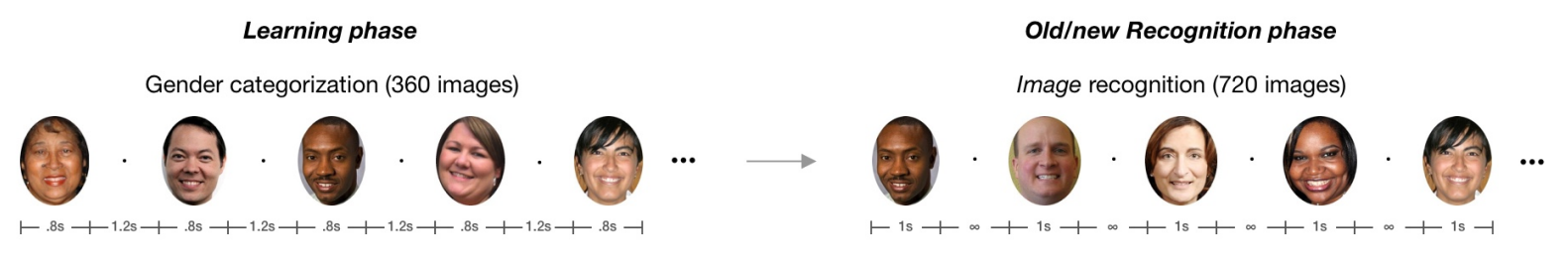

\section{Experiment 2}

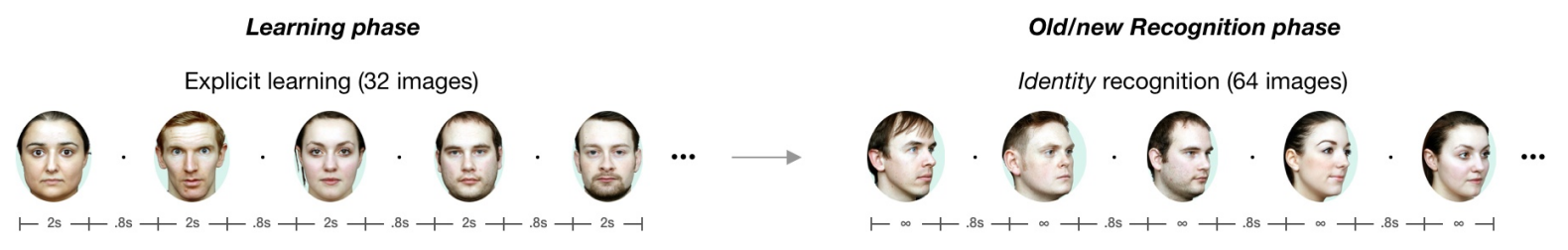

Figure 1. Experimental procedures. In Experiment 1, observers implicitly learned images in the context of a gender categorization (i.e., orthogonal) task. Subsequently they were required to complete a surprise old/new recognition task in which the exact implicitly learned frontal target images (high and low memorability) had to be distinguished among novel distractors (medium memorability). In Experiment 2, observers were explicitly asked to learn a set of (frontal) faces for subsequent recognition. Here, in the recognition phase target identities and novel distractors were presented at 3/4 view.

In Experiment 2, to-be-remembered identities were explicitly learned from frontal view face images (Figure 1, bottom). With the intention of probing memorability and recognition for identity rather than images, the subsequent old-new recognition task involved stimuli shown from a three-quarter viewpoint. Again, we anticipated better recognition across viewpoints for high vs. low memorability identities. Yet here, SRs outperforming controls would indicate that their superior skill is not derived from purely image-dependent information, but instead reflects more efficient extraction of viewpoint-invariant representations of facial identity.

Most importantly, we investigated individual and group performance profiles across experiments. We hypothesized that consistency of memorability-dependent recognition performance observed between experiments indicates utilization of viewpoint-invariant information during recognition. Therefore, greater consistency of recognition performance among SRs vs. typical observers² would imply that SRs extract viewpoint-invariant, identitydiagnostic information from face images in a fundamentally more principled and consistent

2 Systematically varying both the spatial frequency and orientation content of unfamiliar face stimuli processed in an identity matching task, Nador and colleagues (2021; submitted) found that SRs utilize the same range of spatial frequency content across orientations as controls, albeit more consistently. While this study did not explicitly assess memorability, its findings at least suggest that within the sub-process of face perception, controls and SRs vary only quantitatively. 
manner. And, to the extent that performance agrees with image- or identity-based memorability, this would provide evidence for the continuous and dimensional nature of memorability.

We find that, for controls, recognition performance across experiments varies inter-individually, and independently of stimulus memorability. SRs, on the other hand, show significant consistency between their memory performance and average stimulus memorability across experiments. We therefore surmise that SRs have a more concrete dimensional representation of face memorability, untethered to image-specific information available at encoding and retrieval, but better entwined with the aspects of facial identities that render them memorable. This is of particular importance given its implications for diagnosis of pathology (Bainbridge et al., 2019), as well as for identification of potential SRs in future research (Isola et al., 2011; Ramon et al., 2019; Ramon, In press; Ramon et al., 2019) and applied settings (Ramon, in press; Ramon \& Rjosk, in press; Ramon \& Wyss, 2021).

\section{Materials \& Methods}

Observers

Thirty-four observers (26 females), aged 18-47 years ( $M=27.5$; $S D=8.33)$, who were either students at the University of Fribourg or non-student individuals contacted by the experimenters, provided informed consent to participate in both experiments (as approved by the local research ethics committee (approval number 473), adherent to the Declaration of Helsinki). Control observers received course credit or financial compensation for their participation. The SR sample consisted of 11 individuals (all right-handed) who achieved superior performance in at least two of three tests of face perception and recognition (Yearbook Test (YBT; Bruck et al., 1991), Facial Identity Card Sorting Test (FICST; Jenkins et al., 2011), Cambridge Face Memory Test long version (CFMT+; Russell et al., 2009) (see Table 1) derived from a recently reported cohort of 70 SR cases (Ramon, in press). Some of the cases reported here also participated in additional behavioural, oculomotor and neuroimaging studies (Nador et al., Submitted a \& b; Linka et al., 2021; Faghel-Soubeyrand; 2020). One control and one SR observer's data were excluded from both experiments due to chance level performance.

\section{Stimuli}

In Experiment 1, 720 face (half fe/male) stimuli from the 10K US Adult Faces Database (Bainbridge et al., 2012) measuring .53 of visual angle (VA) (1024x768 pixels; $57 \mathrm{~cm}$ viewing distance), labelled with hit rate-derived memorability scores (Bainbridge et al., 2017), and an average contrast of .96 (min= .63, max =.99) were displayed on a MacBook Pro (15", Mid 2010; 2.4 GHz Intel Core i5). Target stimuli comprised 180 high and low memorability images; 360 medium memorability images served as novel distractors for the old/new recognition task. In Experiment 2, 64 face images with hit rate-derived memorability scores (Bainbridge, 2016) originating from the Karolinska Directed Emotion Faces (KDEF) database (Lundqvist et al., 1998) and the Stirling Economic \& Social Research Council (ESRC) 3-Dimensional Face Database (Hancock \& Tiddeman, 2011) were used. Stimuli subtended .53 VA and had an average contrast of .95 (min=.72, max=.99) (see Figure 1). 
Procedures

Each experiment consisted of two phases: first a learning phase, and then a recognition phase. During the learning phase, observers learned (implicitly in Experiment 1; explicitly in Experiment 2) a set of face stimuli and committed them to memory. During the recognition phase, they were tasked with recognizing face stimuli as either matching (exact image matches in Experiment 1; identity matches in Experiment 2) one of the studied identities, or as a novel foil. An equal number of targets and foils were presented to observers in each experiment.

\section{Experiment 1 -Recognizing Implicitly Learned Face Images}

In the implicit learning phase of Experiment 1, observers bimanually categorized high and low memorability stimuli as male ('press A') or female ('press L') as quickly and accurately as possible. Stimulus presentation durations are detailed in Figure 1; responses were recorded during stimulus presentation or the following blank interval. In total, six blocks of 60 images (with interleaved 10s breaks; order randomized) were shown. Then, after a short (3-5min) break, the experimenter indicated that the previous gender categorization task was in fact an implicit learning task and that a recognition would now follow (all observers were unaware of the subsequent recognition task). In the recognition phase, observers were instructed to indicate via button press - as quickly and accurately as possible-whether they had seen each presented image during the learning phase ('A' for 'old'; 'L' for 'new'). This task comprised 4 blocks of 180 stimuli (in randomized order, with self-paced breaks between blocks). Stimuli were presented for 1s, followed by a blank screen that persisted until a response was provided.

\section{Experiment 2 - Recognizing Explicitly Learned Faces across Viewpoint Changes}

After completion of Experiment 1, observers were given another short break (3-5 min), before beginning the explicit learning phase of Experiment 2. During this phase, they were explicitly instructed to learn 32 new faces for subsequent recognition. After a short (30s) break, the recognition phase began. Observers were instructed to indicate as quickly and accurately as possible whether the 3/4 viewpoint face stimuli represented facial identities had been presented in the prior learning phase (i.e. press ' $A$ ' for 'old') or not (i.e. press 'L' for 'new'). The 64 stimuli were presented randomly in a single block; each was displayed until a response was provided and trials were separated by a .8s blank screen ITI.

\section{Results}

Mixed ANOVA

A three-way mixed ANOVA (2 Group $\times 2$ Experiment $\times 2$ Memorability) - with group as the only between-subjects factor - revealed no main effect of Group $(F(1,30)=.502 ; p=.484)$, but main effects of Memorability $(F(1,30)=48.05 ; p<.01)$ and Experiment $(F(1,30)=5.69 ; p=.024)$, indicating comparable performance across groups, but not stimulus memorability or experiments. No Group by Memorability interaction emerged $(F(1,30)=.04 ; p=.83)$, though there was a significant interaction between Memorability and Experiment $(F(1,30)=26.80$; $\mathrm{p}<$.01). Post-hoc t-tests revealed higher hit rates for low-memorability stimuli in Experiment 2 than Experiment $1(t(62)=-2.34 ; p=.022)$, while the hit rate for high-memorability stimuli did not significantly differ across experiments $(t(62)=.455 ; p=.651)$. Furthermore, we noted a significant interaction between Group and Experiment $(F(1,30)=5.93 ; p=.021)$. Post-hoc ttests showed comparable performance for controls across experiments (t(86)=.325; $p=.746$ ), 
while SRs performed significantly better in Experiment 2 than Experiment 1 (t(38)=-2.226; $p=.03)$. There was no evidence of three-way interaction $(F(1,30)=.122 ; p=.730)$.

\section{"With or Without You" (WoWY) Analysis}

In order to assess whether individual observers showed significant sensitivity to memorability in each experiment, we first computed individual WoWY scores following the procedures outlined in Figure 2 (See Supplementary Information for a more detailed description of the methodology). Then, we correlated each individual's WoW scores with average memorability, across all images' memorability rankings. Effectively, this entailed testing the alternative hypothesis that WoWY scores covary with memorability against the null hypothesis that WoW scores are independent of memorability. We then tested the significance of each correlation coefficient by comparing it to the bootstrapped distribution of correlation coefficients obtained by randomly permuting memorability rankings between images (Figure 3).

In Experiment 1, 11 controls showed significant positive correlations between their WoW scores and memorability $(r(358)=.33, .46, .51, .49, .27, .42, .51, .44, .33, .21, .28$; ps<.05), while 5 showed negative correlations $(r(358)=-.33,-.64,-.16,-.66,-.51 ;$ ps<.05), and 6 showed none (see Supporting information for all individual observers' WoWY data). Five SRs showed significant positive correlations $(r(178)=.49, .16, .25, .25, .37$; ps<.05) and 5 showed negative ones $(r(178)=-.29,-.28,-.15,-.66,-.29 ;$ ps<.05). In Experiment 2, only 4 controls showed significant positive correlations $(r(14)=.65, .72, .71, .7, .65$; ps<.05) and just 3 showed negative ones (r(14)=-.64,-.69,- .58). Meanwhile, among SRs, only 2 of the 10 SRs showed significant positive correlations $(r(14)=.53, .93 ; p s<.05)$ and 3 showed negative ones $(r(14)=-.65,-.72, \quad-.30 ;$ ps<.05) (see Figure 3 and Supporting information for individual observers' scatter plots).

Over and above the significance of these correlations, though, our primary interest is in understanding consistency of individuals' performance as a function of memorability between Experiments 1 and 2, as this speaks to whether observers tended to have similar patterns of sensitivity to memorability when diagnostic information was more image-dependent, or viewpoint-invariant. We therefore Z-transformed each observer's abovementioned correlation coefficients relative to the bootstrapped distributions used for their significance testing (Figure 2). Thus, for each observer, we obtain one WoWY Z-score per experiment, reflecting their performance relative to the average, across memorability. As demonstrated in Figure 3, among controls there was no correlation between WoWY Z-scores between Experiments 1 and 2. Among SRs, however, there is a significant positive correlation $(r(8)=.67$, pboot $=.01$ ) when compared to the bootstrapped distribution of correlation coefficients obtained by resampling 10 observers (irrespective of group) at a time (rightmost panel of Figure 3a). ${ }^{3}$

\footnotetext{
3 The decision to resample 10 observers at a time in creating the bootstrapped distribution of correlation coefficients between Zscores arises from the fact that the sample of included SRs in which we found this correlation had $N=10$. Resampling 10 observers at a time thereby ensured that the bootstrapped sampling distribution would have a standard deviation commensurate with the standard error of the mean for an obtained sample of size 10.
} 


\section{"With or without you" (WoWY) procedure implemented in each experiment}
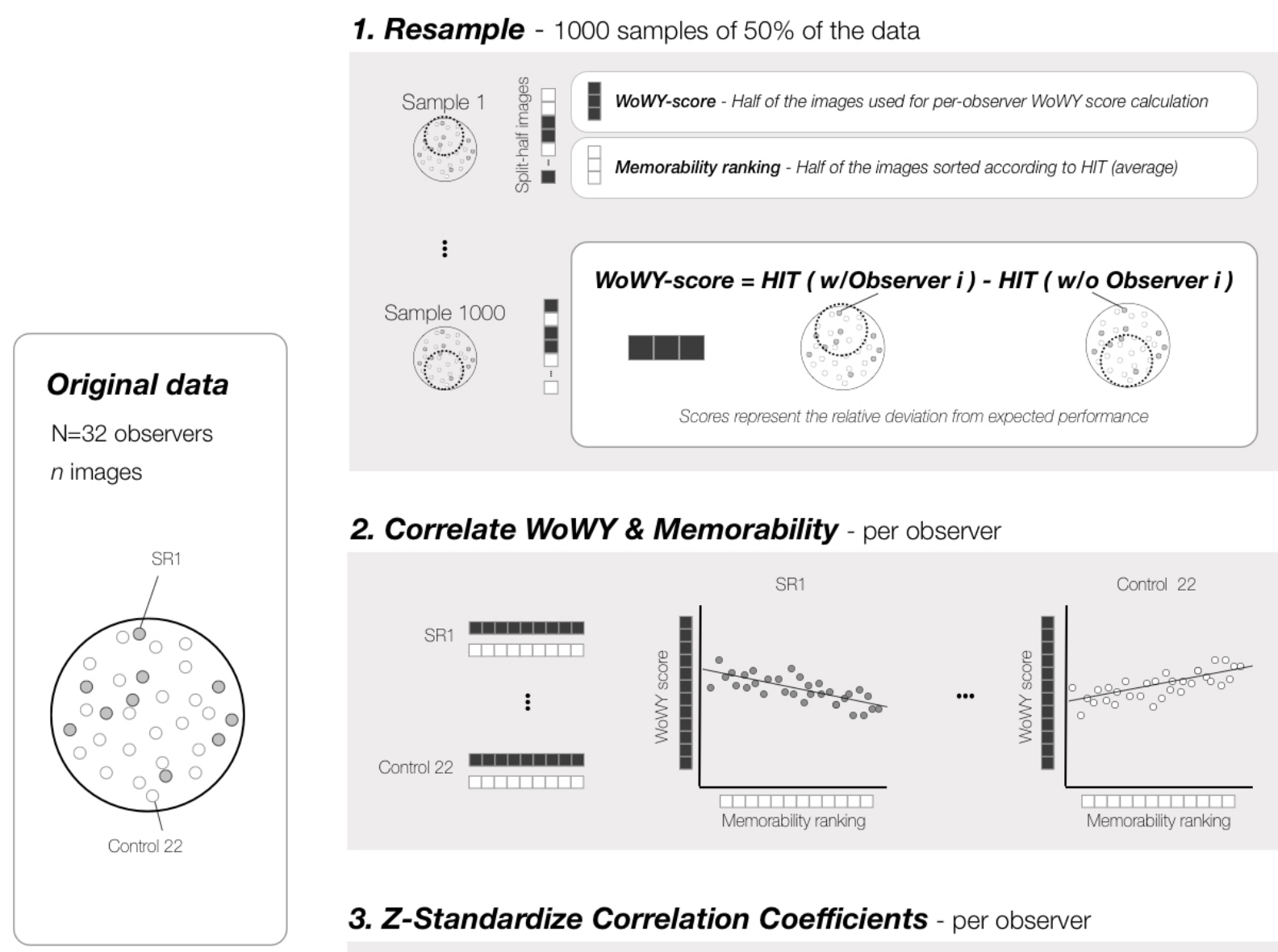

2. Correlate WoWY \& Memorability - per observer

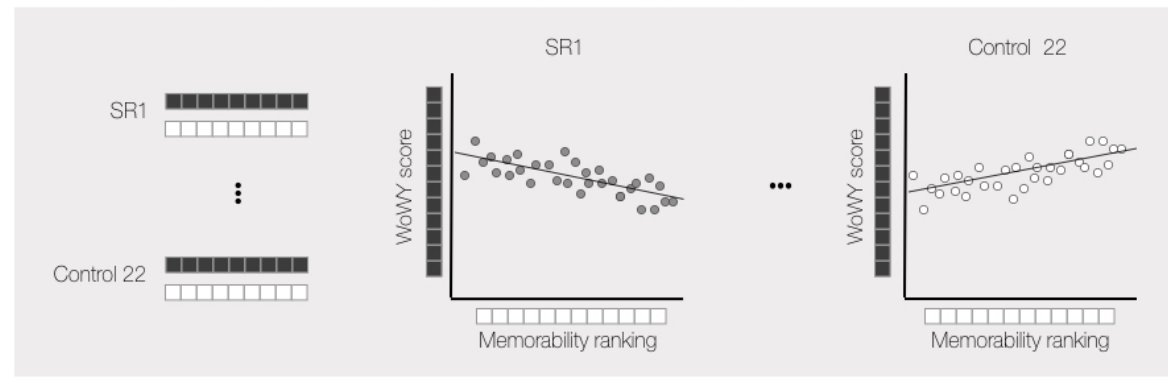

3. Z-Standardize Correlation Coefficients - per observer

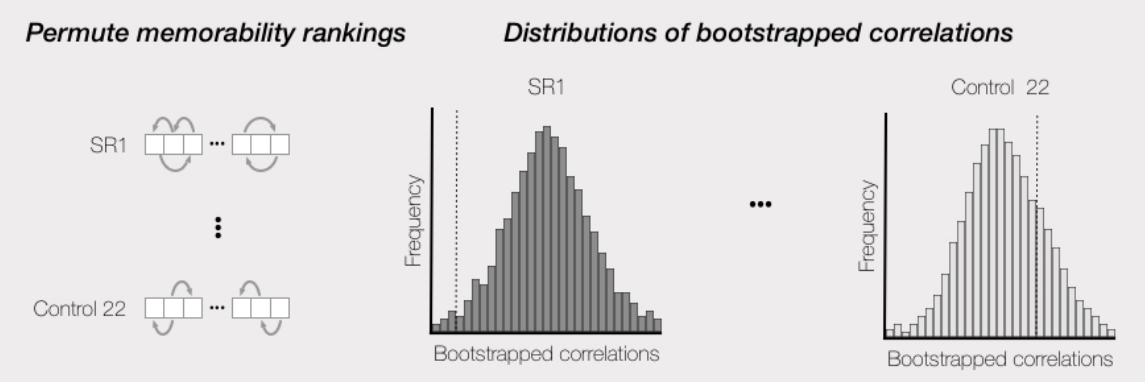

Figure 2. Schematic representation of the "with or without you" (WoWY) analysis. 1. Performance data for half of all images and observers, respectively, were repeatedly resampled (without respect to SR status) and retained for computing WoWY scores; the remaining half were used for computing average memorability. 2. Over repeated samples, each observer's influence on mean performance as a function of memorability could be assessed by correlating their WoWY score against average memorability (derived from all other observers' recognition performance for those images). 3. The impact of memorability on WoW scores was assessed in each experiment by standardizing the correlation coefficients obtained in (2); parameter estimates were derived by repeatedly permuting memorability scores, and assessing the correlation obtained with each new ordering of images. 

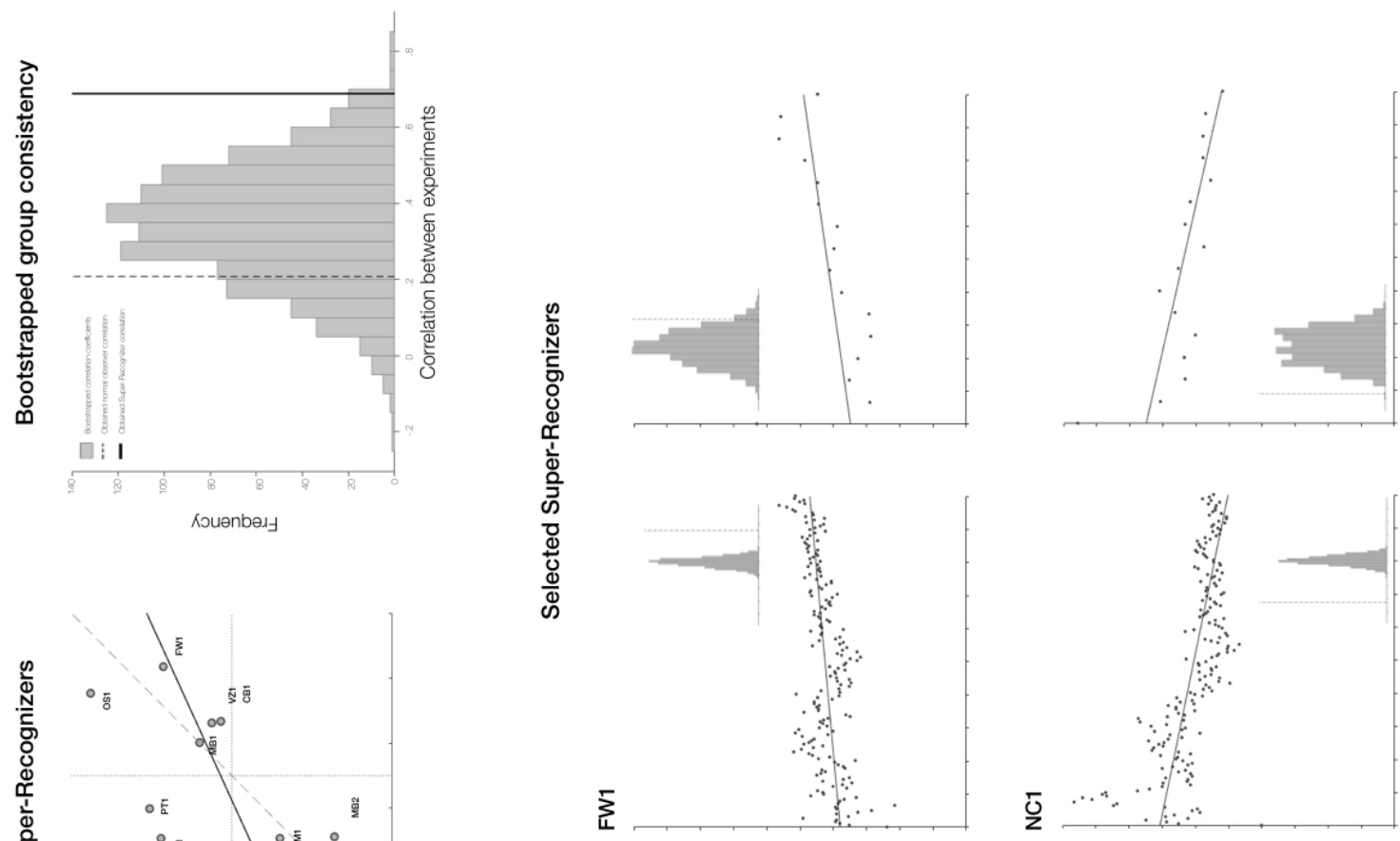

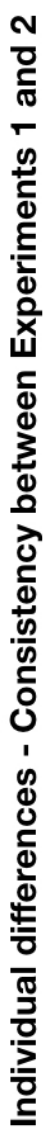
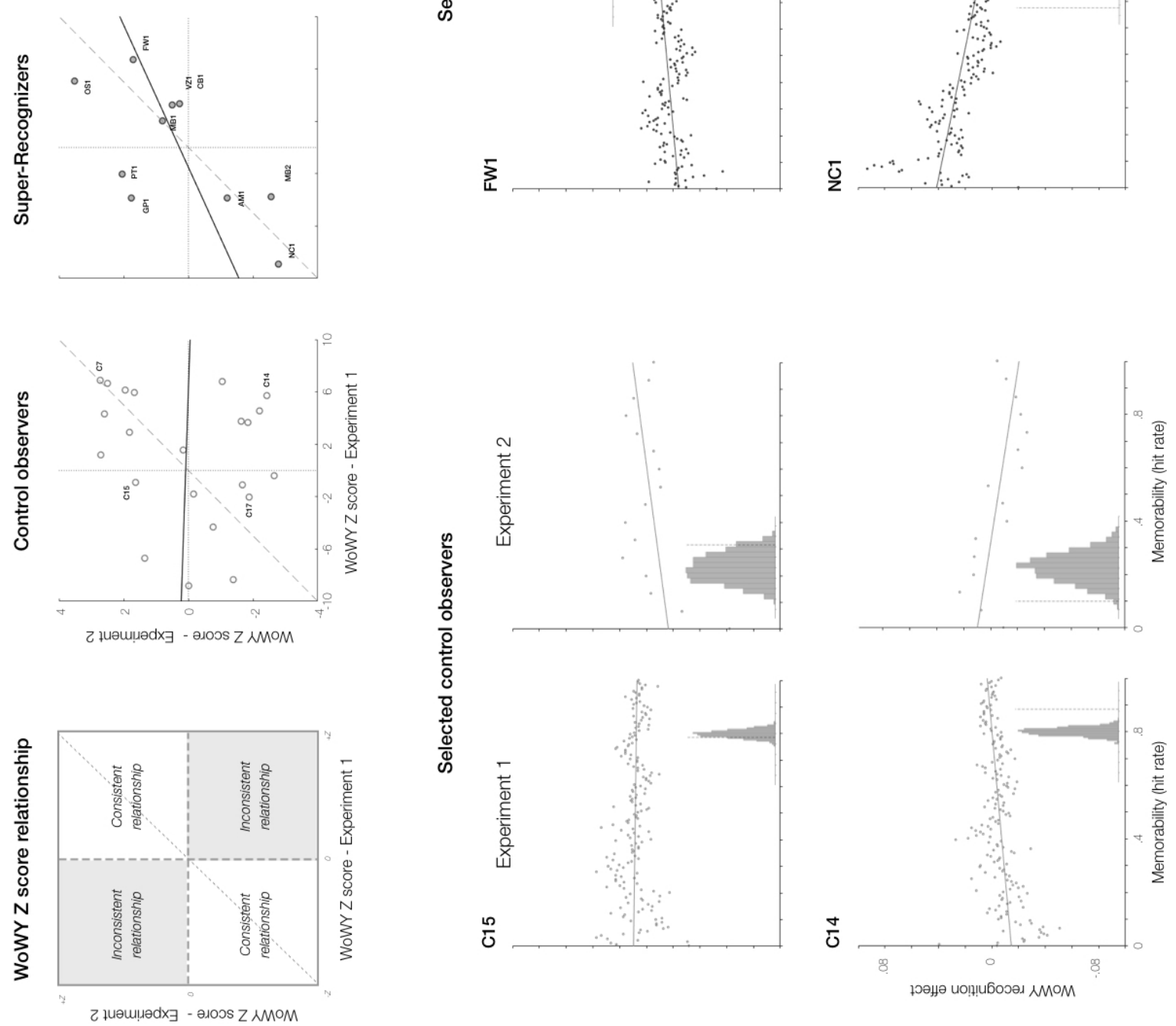

ஸ்

i

Figure 3. a. Correlation between WoWY Z-scores obtained for each observer in Experiments 1 \& 2 . a.

Relationship between WoWY Z-scores. Observers in quadrants 1 \& 3 have consistent representations of memorability across experiments, while those in quadrants 2 \& 4 have inconsistent representations. Deviation of WoWY Z-scores from zero indicates the observer's within-experiment agreement between recognition performance and memorability (center). Significance of correlation coefficient assessed by bootstrap (right). b. WoWY Z-scores for a representative subset of controls (left) and SRs (right). The scatter plots display correlations between the observer's WoWY scores and memorability. Inset histograms show the bootstrapped distributions of correlation coefficients obtained by permuting memorability ordering. Positive correlation indicates increasing recognition performance relative to peers as a function of memorability, and negative correlation indicates decreasing recognition memory performance relative to peers as a function of memorability. All observers' scatter plots and histograms are provided in the Supporting information. 


\section{Discussion}

Across our two experiments, we find that recognition performance varies as a function of memorability scores, which were derived from independent observers' recognition performance measured in the context of different paradigms (Bainbridge et al., 2013; Bainbridge et al., 2017; Bainbridge, 2017; Bainbridge, 2020). As well, SRs did not outperform controls at the group level, suggesting that any average recognition memory differences between groups were modest at best. Given that both groups show comparable memorability-dependent performance, our results lend credence to the notion that imagebased memorability is consistent across samples and experimental paradigms. Though of course, the fact that we cannot derive an identity-based effect between our studies and those of others (Bainbridge, 2017; Bainbridge et al., 2017) does not preclude its existence altogether. Indeed, we do find an experiment by memorability interaction, suggesting that image- and identity-based (i.e., viewpoint-invariant) memorability effects might be separable. However, the studied identities (and their memorabilities) themselves differed between experiments, and so this hypothesis cannot be adjudicated by ANOVA alone.

To wit, ANOVA revealed no group by memorability interactions, suggesting that SRs were no better than normal counterparts at recognizing either low or high memorability faces. In this particular case, however, the absence of evidence should not be taken for evidence of absence. For instance, the high and low memorabilities tested here could have either been too extreme or too heterogeneous. Extreme low and high memorability conditions could have resulted in floor and ceiling performance, respectively, though this seems unlikely, given the observed memorability by experiment interaction (cf. ROC Analysis in the Supplementary Information). In fact, recent work by Bainbridge (2020), using a very similar paradigm to the current experiments', finds comparable levels of performance. Consequently, our high and low memorability stimuli seem neither too heterogeneous, nor too extreme, which points to an effect of information content (image-dependent versus viewpoint-invariant) on memorability, as it did, after all, have a main effect on performance.

While, in Experiment 1, SRs' average performance may not have been substantially superior to normal counterparts, they did seem to exhibit enhanced sensitivity to viewpoint-invariant information diagnostic of identity, as revealed by individualized WoW analyses. That is, while overall performance might have been similar between groups, more granular analyses revealed that SRs were more attuned to identity-based memorability: across relatively large changes in viewpoint, SRs tended to forget forgettable and recognize memorable identities with greater facility than controls did. From this, we might expect any given normal observer to have a more idiosyncratic memorability profile (i.e., recognition for specific identities) that is less consistent with that of other observers. In order to determine whether this was the case, we assessed the diagnosticity of image-dependent versus viewpoint-invariant information by determining memorability in terms of the marginal performance across observers, separately for each image (and for each observer).

In Experiment 1, split-half WoWY analysis showed that observers' performance was more consistent with memorability across images (derived from other observers' performance) than would be predicted by chance alone (Figure 3), such that individual observers' performance would show no correlation with permuted (and thus disordered) image memorability scores. In all but 6 observers, though, we find there were significant deviations from chance in Experiment 1, indicating that the ordering of stimulus memorability (ranked based on all other 
observers' performance) was significantly correlated with their own performance. In line with previous research, this result strongly suggests that stimulus memorability is consistent across observers (Bainbridge et al., 2013; Chang et al., 2017). However, recall that in Experiment 1 observers learned targets (face images) implicitly in the context of an orthogonal (gender categorization) task, and their recognition performance for these targets was tested using these exact same images. Thus, from these results alone, it is unclear whether the internal consistency of memorability was derived from image-dependent or viewpoint-invariant identity diagnostic information, since either could have been successfully used to aid recognition of an exact image match during the recognition phase. Note however, that consistency between hit rates in Experiments 1 and 2 would only be expected if viewpoint-invariant information were used in both, since image-dependent information could not.

In Experiment 2, memorability of the to-be-learned target identities varied continuously (c.f. Bainbridge, 2016), whereas the images used in Experiment 1 were taken from the high and low memorability tails of a performance distribution (c.f. Bainbridge et al., 2017). Therefore, by comparison with Experiment 1, the correlation between observers' WOWY performance and stimulus memorability was weaker within observers, on average. But crucially, since observers' explicit recognition was based on differing viewpoints of the studied identities, their performance required viewpoint-invariant identity (as opposed to image) recognition. While observers explicitly encoded faces for later recognition, the instructions provided no indication as to whether or not identical images would be used to measure subsequent performance. Under these circumstances, local features would be exceedingly unlikely to transfer from head-on to three-quarter viewpoints unless an observer utilized some viewpoint-invariant information.

Split-half permutation analysis confirms this, but also shows significantly greater consistency with the results of Experiment 1 among SRs than controls (Figure 3). More specifically, SRs tended to exhibit consistent patterns of recognition performance (across experiments) as a function of memorability, whereas controls did not. Overall, this supports the hypothesis that SRs show higher sensitivity to viewpoint-invariant information as a continuous dimension. Furthermore, it implies that compared to controls, SRs more consistently built holistic and robust representations of encoded facial identities across experiments - even without explicit instructions to do so (i.e., Implicit Learning Phase, Experiment 1).

When we surreptitiously solicited stimulus memory after encoding during a gender categorization task, SRs' recognition performance was consistent with the formation of viewpoint-invariant, memorability-dependent identity representations, whereas controls were equally likely to form image-specific face representations instead. This can be seen by the distribution of controls' WoWY Z-scores across all four quadrants of Figure 3: none of the SRs who showed memorability-dependent performance in Experiment 2 also showed memorability-dependent performance inconsistent with Experiment 1. They are thus overrepresented in quadrants 1 and 3 of Figure 3, whereas a large proportion of controls can be found in quadrant 4 (indicating inconsistent performance).

This is indicative of an important-and seemingly qualitative-distinction between SRs' and controls' internal concepts of memorability as a dimension of face stimuli: SRs seem to implicitly build viewpoint-invariant representations of facial identity, rather than relying on image-dependent information. In line with this hypothesis, the split-half permutation test 
revealed that both SRs and controls use stimulus features reliably aligned with memorability in Experiment 1, wherein target stimuli shown during the recognition phase were identical to the stimuli shown during the implicit learning phase. Yet critically, only among SRs do we note a significant positive correlation between information usage in Experiments 1 and 2, implying that this information was most likely viewpoint-invariant.

SRs' consistency between image- and identity-based memorability is qualitatively unlike that of controls. Interestingly, when it comes to face memory, there tends to be broad agreement between individuals about which faces they are most likely to recognize (Isola et al., 2011; Bainbridge et al., 2013; Bainbridge, 2017). Our results generally agree with these previous findings. Herein, when faces' memorabilities were measured according to all but one observer's performance, that remaining observer's WoWY scores indicate clear covariation with others' abilities to remember images of similar memorability. Moreover, this effect was much larger when recognition was probed in an image-dependent (Experiment 1), as opposed to viewpoint-invariant (Experiment 2) manner. Taken together, our results strongly suggest that face memorability can be conceptualized as a viewpoint-invariant and dimensional attribute, to which at least SRs seem to be highly sensitive. In the context of previous results, this could mean that internal agreement between image- and identity-based memorability may be lower or higher depending on the pictorial similarity of stimuli used during encoding/learning and recognition.

Here, since face recognition was probed under relatively extreme circumstances, we cannot exclude the possibility that controls might indeed be able to build representations resembling those of SRs if given longer (or more frequent) exposure to face stimuli during learning, or less extreme viewpoint changes during recognition. While here we do find strikingly different patterns of recognition performance between controls and SRs related to memorability, this does not provide unequivocal evidence for either qualitative or quantitative differences between groups.

Similarly, face recognition memory relates to memorability in a qualitatively different way (and more strongly) than it does to attentional or priming processes that occur during encoding (Bainbridge, 2020). So, it remains a distinct possibility that the specific depth(s) of encoding actually used by controls might produce more or less viewpoint-invariant memorial representations. Here, however, we tested observers only at medium and high depths of encoding. At the opposite end of the spectrum, we could consider testing SRs at very low depths of encoding (e.g., with only passive viewing during the learning phase) to see whether their ability to extract viewpoint-invariant identity information from face images would persist. And critically, the effect of the specific learning phase tasks has not been investigated in the context of identity-based memorability, but only exact image matches. Thus, whether memorability for identities varies as a function of attention or priming remains an open question, also amenable to scrutiny under an expanded set of stimulus and instruction conditions.

\section{Conclusion}

In sum, we propose that face memorability should not only be conceptualized in terms of memory for a specific image (e.g., Bainbridge et al., 2013; Khosla et al., 2015; Broers et al., 2017), but also with respect to viewpoint-invariant information diagnostic of its identity (e.g., Bartlett et al., 1984; Bruce et al., 1984; Valentine et al., 2004; Bainbridge, 2017; Chang et 
al., 2017). Our results thus invite a reinterpretation of face memorability to include identitydiagnostic information conveyed across varied viewpoints, as well as a more detailed evaluation of the content of such representations. Currently, it remains relatively unclear what kind(s) of information (i.e., image statistics) contained across various viewpoints of a given facial identity are in fact crucial for its memorial representation. Future research should consider the use of SRs as a special population particularly adept at this ability, and comparison with controls who may well also be capable under less straining stimulus conditions, as well as automatic solutions developed for face recognition.

\section{Author Contributions}

MR \& TAA designed the experiments; TAA acquired the data; JDN \& AG Analyzed the data; MR, JDN \& AG Wrote the Manuscript.

\section{Acknowledgements}

We thank all observers for their participation, Matteo Zoia for assistance during data acquisition, and Wilma Bainbridge for providing information and stimulus material. MR is supported by a Swiss National Science Foundation PRIMA (Promoting Women in Academia) grant (PROOP1_179872).

\section{Conflict of interest statement}

The authors declare no conflict of interest.

\section{Data Accessibility Statement}

All raw data and code for the WoW analyses will be made publicly available upon publication on the Open Science Framework (https://osf.io/5p7yk/).

\section{References}

Bainbridge, W. A. (2017). The memorability of people: Intrinsic memorability across transformations of a person's face. Journal of Experimental Psychology: Learning, Memory, and Cognition, 43(5), 706-716. doi:10.1037/xIm0000339

Bainbridge, W. A. (2020). The resiliency of image memorability: A predictor of memory separate from attention and priming. Neuropsychologia, 141, 107408. doi:10.1016/ j.neuropsychologia.2020.107408

Bainbridge, W. A., Berron, D., Schütze, H., Cardenas-Blanco, A., Metzger, C., Dobisch, L., . . . Düzel, E. (2019). Memorability of photographs in subjective cognitive decline and mild cognitive impairment: Implications for cognitive assessment. Alzheimer's \& Dementia: Diagnosis, Assessment \& Disease Monitoring, 11(1), 610-618. doi:10.1016/ j.dadm.2019.07.005

Bainbridge, W. A., Dilks, D. D., \& Oliva, A. (2017). Memorability: A stimulus-driven perceptual neural signature distinctive from memory. Neurolmage, 149, 141-152. doi:10.1016/ j.neuroimage.2017.01.063

Bainbridge, W., Isola, P., Blank, I., \& Oliva, A. (2012). Establishing a database for studying human face photograph memory. In Proceedings of the Annual Meeting of the Cognitive Science Society (Vol. 34, No. 34).https://escholarship.org/uc/item/49p3934p

Bainbridge, W. A., Isola, P., \& Oliva, A. (2013). The intrinsic memorability of face photographs. Journal of Experimental Psychology: General, 142(4), 1323-1334. doi:10.1037/a0033872 
Bartlett, J. C., Hurry, S., \& Thorley, W. (1984). Typicality and familiarity of faces. Memory \& Cognition, 12(3), 219-228. doi:10.3758/bf03197669

Bobak, A. K., Mileva, V. R., \& Hancock, P. J. (2018). Facing the facts: Naive participants have only moderate insight into their face recognition and face perception abilities. Quarterly Journal of Experimental Psychology, 72(4), 872-881. doi:10.1177/1747021818776145

Broers, N., Potter, M. C., \& Nieuwenstein, M. R. (2017). Enhanced recognition of memorable pictures in ultra-fast RSVP. Psychonomic Bulletin \& Review, 25(3), 1080-1086. doi:10.3758/s13423-017-1295-7

Bruce, V., Burton, M. A., \& Dench, N. (1994). What's Distinctive about a Distinctive Face? The Quarterly Journal of Experimental Psychology Section A, 47(1), 119-141. doi:10.1080/14640749408401146

Bruck, M., Cavanagh, P., \& Ceci, S. J. (1991). Fortysomething: Recognizing faces at one's 25th reunion. Memory \& Cognition, 19(3), 221-228. doi:10.3758/bf03211146

Chang, C., Nemrodov, D., Lee, A. C., \& Nestor, A. (2017). Memory and Perception-based Facial Image Reconstruction. Scientific Reports, 7(1). doi:10.1038/s41598-017-06585-2

Faghel-Soubeyrand, S., Ramon, M., Bamps, E., Zoia, M., Woodhams, J., Alink, A., . . . Charest, I. (2020). Multivariate pattern analysis reveals domain-general enhancement of visual representations in individuals with "super-recognition" of faces. Journal of Vision, 20(11), 502. doi:10.1167/jov.20.11.502

Fysh, M. C., Stacchi, L., \& Ramon, M. (2020). Differences between and within individuals, and subprocesses of face cognition: Implications for theory, research and personnel selection. Royal Society Open Science, 7(9), 200233. doi:10.1098/rsos.200233

Hancock, P. J. B., \& Tiddeman, B. (2011). Stirling/ESRC 3D Face Database. http:// pics.stir.ac.uk/ESRC/3d images.htm

Isola, P., Parikh, D., Torralba, A., \& Oliva, A. (2011). Understanding the Intrinsic Memorability of Images, doi:10.21236/ada554133

Isola, P., Xiao, J., Torralba, A., \& Oliva, A. (2011). What makes an image memorable? Cvpr 2011. doi:10.1109/cvpr.2011.5995721

Jenkins, R., White, D., Montfort, X. V., \& Burton, A. M. (2011). Variability in photos of the same face. Cognition, 121(3), 313-323. doi:10.1016/j.cognition.2011.08.001

Khosla, A., Raju, A. S., Torralba, A., \& Oliva, A. (2015). Understanding and Predicting Image Memorability at a Large Scale. 2015 IEEE International Conference on Computer Vision (ICCV). doi:10.1109/iccv.2015.275

Linka, M., Alsheimer, T., Broda, M. D., de Haas, B., \& Ramon, M. (Mar. 14-17, 2021). Atypical Visual Salience in Super-Recognizers. Tagung experimentell arbeitender Psychologen (Conference of Experimental Psychologists): Ulm, Germany.

Lundqvist, D., Flykt, A., \& Öhman, A. (1998). The Karolinska directed emotional faces (KDEF). CD ROM from Department of Clinical Neuroscience, Psychology section, Karolinska Institutet, 91(630), 2-2. https://www.kdef.se/home/aboutKDEF.html

Nador, J. D., Zoia, M., Pachai, M. V., \& Ramon, M. (submitted). Super-Recognizers: More Consistent or Qualitatively Different Psychophysical Profiles? 
Nador, J. D., Zoia, M., Pachai, M. V., \& Ramon, M. (Mar. 14-17, 2021). Super-Recognizers: Psychophysical Examination of Individual Differences. Tagung Experimentell Arbeitender Psychologen (Conference of Experimental Psychologists): Ulm, Germany.

Ramon, M., \& Gobbini, M. I. (2017). Familiarity matters: A review on prioritized processing of personally familiar faces. Visual Cognition, 26(3), 179-195. doi:10.1080/13506285.2017.1405134

Ramon, M. (2021). Super-Recognizers -a novel diagnostic framework, 70 cases, and guidelines for future work. Neuropsychologia, 107809. doi:10.1016/ j.neuropsychologia.2021.107809

Ramon, M., Bobak, A. K., \& White, D. (2019). Super-recognizers: From the lab to the world and back again. British Journal of Psychology, 110(3), 461-479. doi:10.1111/bjop.12368

Ramon, M., Bobak, A. K., \& White, D. (2019). Towards a 'manifesto' for super-recognizer research. British Journal of Psychology, 110(3), 495-498. doi:10.1111/bjop.12411

Ramon, M. (2018). The power of how-lessons learned from neuropsychology and face processing. Cognitive Neuropsychology, 35(1-2), $83-86$. doi:10.1080/02643294.2017.1414777

Ramon, M., \& Rjosk, S. (in press). Super-Recognizer in der Polizeiarbeit - Best Practices aus der Entwicklung des "Berlin Model for Super-Recognizer Identification" (BeMo SR-id). Best Practice Handbook of the EU-Project SafeCi - Safer Space for Safer Cities.

Ramon, M., \& Wyss, L. (2021). Super-Recognizers as an example for innovation in policing through science. [Super-Recognizer als Beispiel für polizeiliche Innovation durch Wissenschaft.] Format Magazine - Zeitschrift für Polizeiausbildung und Polizeiforschung | Revue de formation et de recherche policières.

Rugo, K. F., Tamler, K. N., Woodman, G. F., \& Maxcey, A. M. (2017). Recognition-induced forgetting of faces in visual long-term memory. Attention, Perception, \& Psychophysics, 79(7), 1878-1885. doi:10.3758/s13414-017-1419-1

Russell, R., Duchaine, B., \& Nakayama, K. (2009). Super-recognizers: People with extraordinary face recognition ability. Psychonomic Bulletin \& Review, 16(2), 252-257. doi:10.3758/pbr.16.2.252

Russell, R., Duchaine, B., \& Nakayama, K. (2009). Super-recognizers: People with extraordinary face recognition ability. Psychonomic Bulletin \& Review, 16(2), 252-257. doi:10.3758/pbr.16.2.252

Rust, N. C., \& Mehrpour, V. (2020). Understanding Image Memorability. Trends in Cognitive Sciences, 24(7), 557-568. doi:10.1016/j.tics.2020.04.001

Stacchi, L., Huguenin-Elie, E., Caldara, R., \& Ramon, M. (2020). Normative data for two challenging tests of face matching under ecological conditions. Cognitive Research: Principles and Implications, 5(1). doi:10.1186/s41235-019-0205-0

Valentine, T., Darling, S., \& Donnelly, M. (2004). Why are average faces attractive? The effect of view and averageness on the attractiveness of female faces. Psychonomic Bulletin \& Review, 11(3), 482-487. doi:10.3758/bf03196599

Vokey, J. R., \& Read, J. D. (1992). Familiarity, memorability, and the effect of typicality on the recognition of faces. Memory \& Cognition, 20(3), 291-302. doi:10.3758/bf03199666 


\section{Supplemental Material}

Details of the "With or without you" (WoWY) Analysis

To assess whether any individuals' recognition performance was dependent on image- or identity-based memorability, we conducted a split-half, with-or-without-you (WoWM) analysis on each observer's hits and misses. This analysis, illustrated in Figure 2, compares each observer's performance against the null hypothesis that their recall rates across images are independent of image memorability.

The procedure first involves randomly sampling half of the observers and half of the images/ trials (here 1000 samples are drawn, without replacement), whereby observers' and images' expected inclusion rates across samples are 50\%. The drawn samples are inherently divisible into those that include ("with") versus exclude ("without") each observer, or image (Figure 2, Panel 1). Subtracting the hit rates of samples "without" a particular observer from those "with" that same observer yields the relative deviation (WoWY score) from the expected performance across all other observers (i.e., memorability). Next, each observer's WoWY scores (on included images) are ordered according to the memorability of the images from which they were derived. And finally, the sorted WoWY scores are Spearman correlated with the average scores of excluded images across all other observers (also sorted by memorability).

The WoWY analysis' results (a Spearman correlation coefficient) describe the extent to which each observer's performance across images depends on memorability (as assessed by all other observers' recall of the remaining images). In other words, if the recall rates of images across observers were not related to one another, observers' deviations from average memorability (i.e., their WoWY scores) would be distributed randomly. Conversely, if an image is consistently remembered, WoWY scores should approach zero as images are more consistently remembered, and become larger (in magnitude, irrespective of sign) as images are less consistently remembered. For instance, if an observer had a hit unlikely to occur in random samples (i.e., for an image with low memorability), this would produce a large positive WoWY score, and where a hit was quite likely across random samples (i.e., at high memorability), would produce a WoWY score near zero instead. Thus, only if a particular observer's hits and misses are distributed according to image memorability should comparison of their performance with others' result in non-zero Spearman correlation. If their hits and misses were instead distributed randomly across image memorability, we would expect a Spearman correlation coefficient near zero.

To infer whether the null hypothesis (that each observer's Spearman correlation coefficient is effectively equal to zero, regardless of image memorability) should be accepted or rejected, we derived the null distribution from the original memorability-ordered sample by bootstrapping. To that end, we repeatedly sampled Spearman correlation coefficients by permuting the order of images. We could then estimate the likelihood of the originally obtained Spearman correlation coefficient, given the distribution of coefficients generated by perturbing memorability ordering. If the memorability-ordered distribution indeed came from the same population as the permuted ones, its magnitude should occur relatively frequently in the bootstrapped distribution. If, on the other hand, its magnitude were extremely unlikely in the bootstrapped null distribution, we would reject the null hypothesis that the particular observer's recall rates across images was independent of image memorability. 
Individual observer profiles

In addition to the group-level analyses we provide in our manuscript, we here provide more detailed profiles of individual observers' face recognition performance across the two Experiments discussed in the main text. In particular, this section offers an in-depth look at the correlation between observers' WoW scores (see With-or-Without-You Analysis in the Methods section of the main text) and images' average memorability for experiment separately.

In Experiment 1, observers learned implicitly, during completion of a gender discrimination task. Afterwards, they were shown the exact target images implicitly learned, and an equal number of un-studied novel distractor images. In Experiment 2, bservers explicitly learned a series of faces for subsequent recognition (identical to Experiment 1). Here, however, instead of showing them the exact same target images during the recognition phase, we showed them images taken from a different (3/4) viewpoint among foils of the same orientation).

Under these circumstances, in order for an observer to recognize a given face, they would need to extract viewpoint-invariant features of the faces. As such, we would expect an observer's performance to exceed their peers' as they increasingly employ viewpoint-invariant facial information in their representation, particularly in Experiment 2. So, if their performance in both experiments follows a similar pattern, we would infer that they are using similar features for encoding faces when the viewpoints were identical as when they varied. Otherwise, we would surmise that they employed viewpoint-invariant facial information inconsistently between experiments.

The scatter plots in Figure S1 show the correlation between WoWY scores and memorability. As described in the Methods, these are generated for each observer by taking repeated samples of half of all observers (and half of all images) at a time, and then taking the difference in average hit rates between samples including versus excluding that particular observer. We then correlate the two halves: one observer's WoW scores with average memorability for the remaining observers. The histograms corresponding to each plot show the distribution of correlation coefficients that would arise by chance alone-if the ordering of images according to memorability was unrelated to WoWY scores. 


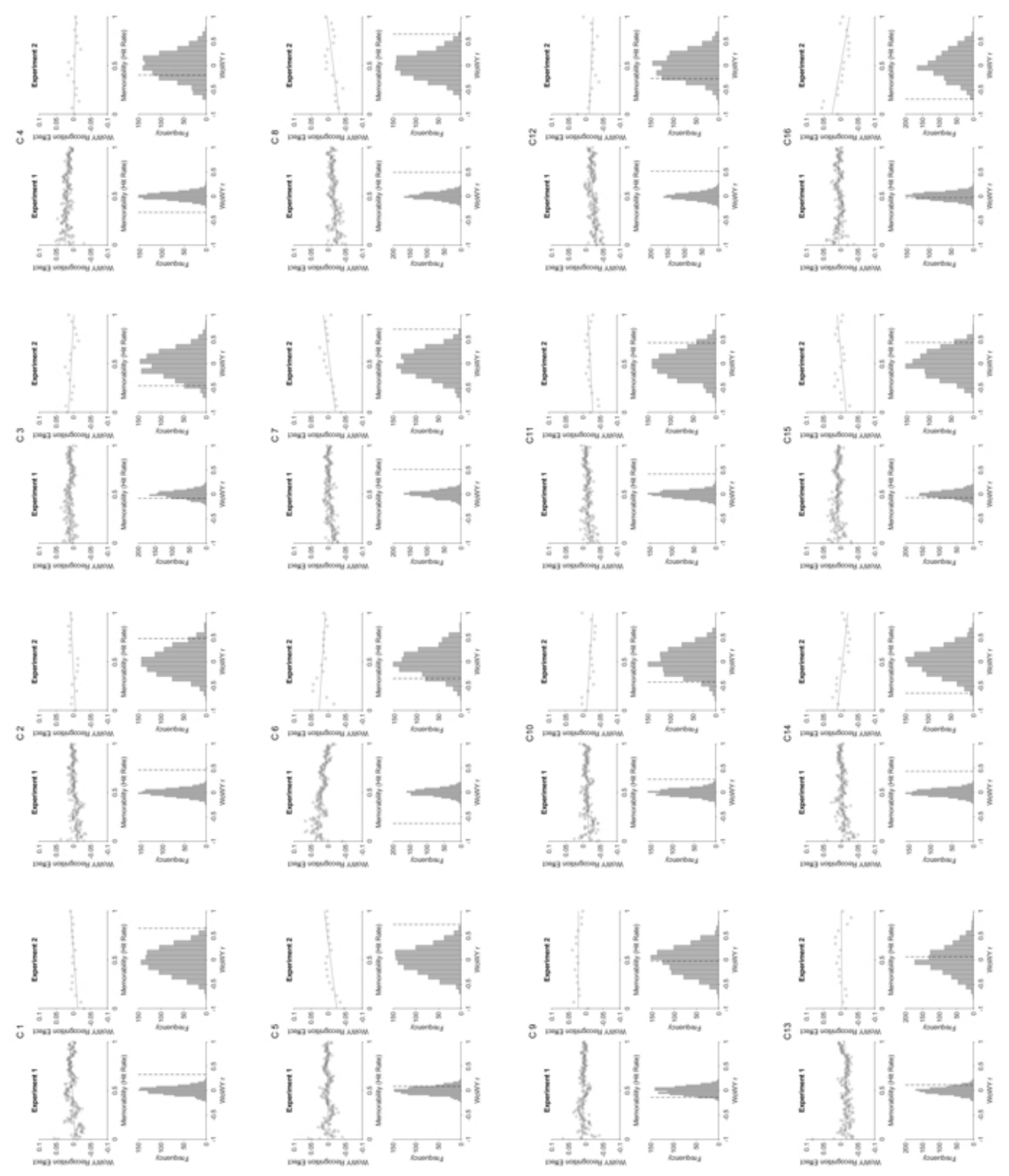

Figure S1a. Scatter plots of individual WoWY scores against average memorability (across all other observers). Histograms display bootstrapped correlation coefficients obtained for permuted (disordered) memorability; dashed lines represent the obtained sample, with memorability order preserved. Shown here are control observers C1-C16. 

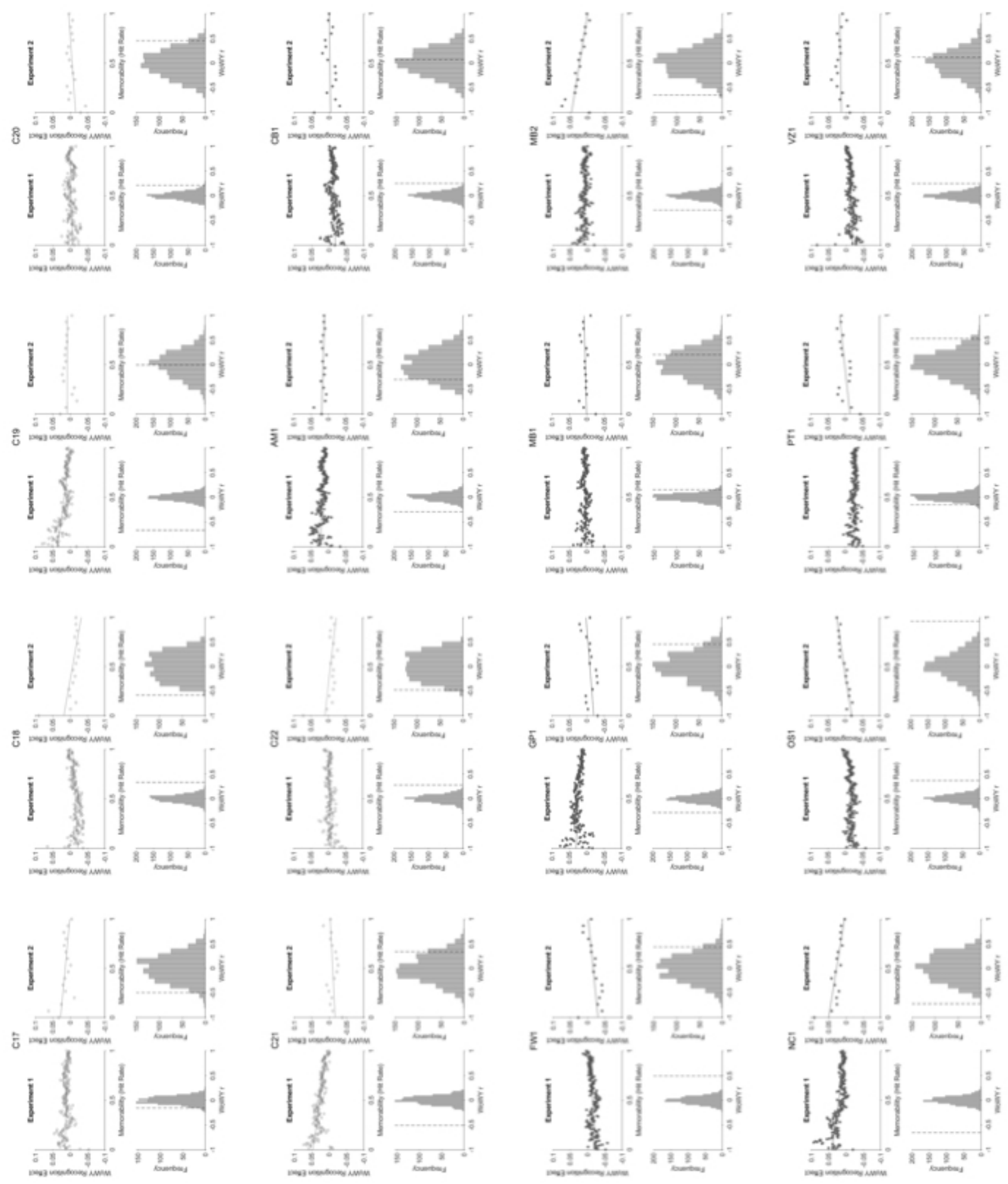

Figure S1b. Scatter plots of individual observers' WoWY scores against average memorability (across all other observers). Histograms display bootstrapped correlation coefficients obtained for permuted (disordered) memorability; dashed lines represent the obtained sample, with memorability order preserved. Shown here are control observers C17-C22 and the 10 SRs. 
Receiver Operating Characteristic Analysis

As the ANOVA results revealed differences in terms of hit rates across the different conditions, we conducted exploratory ROC analyses comparing the proportion of hits and false alarms for high and low memorability stimuli, in both groups and across both experiments to distinguish changes in sensitivity from changes in criterion (see Figure S2). First, we took the mean performance of each observer, separated in terms of hit rates and false alarm rates across low and high memorability stimuli. Then, with the average across observers we generated a group average for each condition. For Experiment 2, as stimuli were continuously rated, we separated them by categorizing half of them as high-memorability and the other half as lowmemorability according to their memorability score. In Experiment 1, normal observers had a slightly higher hit rate as well as a higher false alarm rate than SRs for both low and high memorability stimuli. This indicates criterion differences between groups, with SRs being more conservative than normal observers. In Experiment 2, normal observers and SRs had equivalent false alarm rates, regardless of memorability, yet hit rates among SRs was higher than normal observers' hit rate. This suggests differences regarding sensitivity across groups, with SRs more efficiently distinguishing between previously learned target identities from novel distractors compared to normal observers.
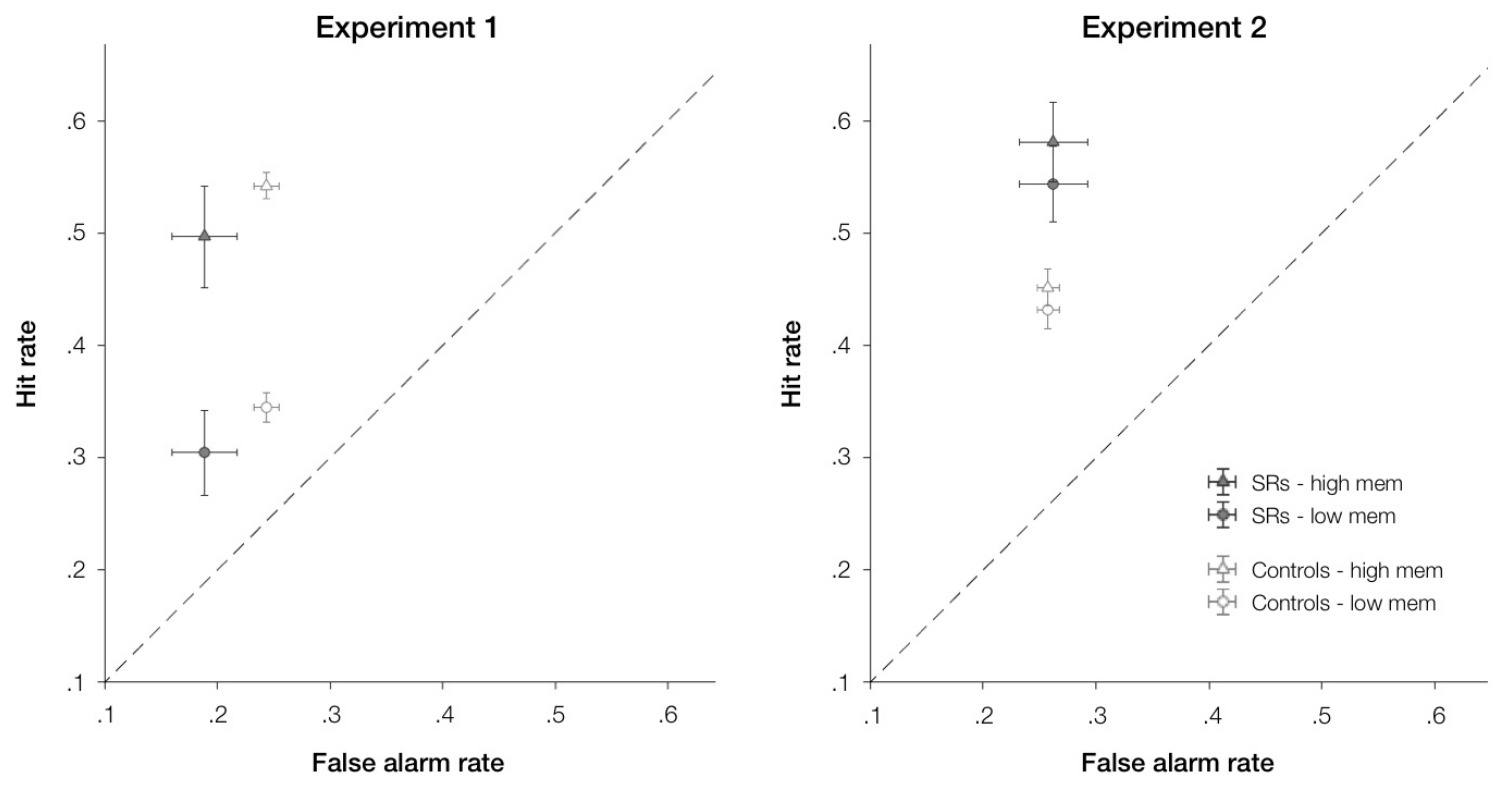

Figure S2. ROC of Hits and False Alarms rates (across Memorability and Group for Experiments 1 and Experiment 2). Points represent the mean hit and false alarm rates of each group; error bars represent 95\% confidence intervals. We note a difference of criterion in Experiment 1, with higher hit rates as well as a higher false alarm rates for normal observers compared to SRs. In experiment 2 we observe a change in sensitivity between groups: while both exhibited comparable false alarm rates, SRs obtained more hits than normal observers. 